\title{
Examining the Interrelationships between Motivation, Conscientiousness, and Individual Endurance Sport Performance
}

\author{
Joanne Perry, Michael Ross, Jeremiah Weinstock and Jeffrey Gfeller \\ Department of Psychology, Saint Louis University, St. Louis, Missouri 63108, USA
}

\begin{abstract}
Personality traits and motivation have been predictive of athletic success. Conscientiousness, intrinsic motivation (IM), and extrinsic motivation (EM) positively predict sport performance, while amotivation (AM) has a negative impact. This study examined the effects of both sport motivation and conscientiousness on athletic performance. Methods included a cross-sectional survey study of endurance athletes $(N=73)$; including runners $(56.1 \%)$, swimmers $(19.2 \%)$, triathletes $(6.8 \%)$, rowers $(2.7 \%)$, and multi-sport athletes (15.1\%). Conscientiousness and motivation were assessed using questionnaires. Subjective ratings and objective scores of a recent performance(s) were collected. Regression analyses demonstrated that conscientiousness positively predicted IM and negatively predicted AM. AM negatively predicted subjective performance. IM and EM were not significant predictors of subjective performance. No variable significantly predicted objective performance. Results support the deleterious role of AM in performance. Compared to IM and EM, AM might be a more relevant construct when predicting performance in a sample of athletes with varying degrees of commitment. This construct may be of particular interest to clinicians due to its association with athletic burnout. Conscientiousness may serve as a protective factor against burnout.
\end{abstract}

Key words: Conscientiousness, motivation, athletic performance, athletic burnout.

\section{Introduction}

Understanding the relationship between personality traits, individual difference variables, and athletic performance is important for predicting athletic success and can provide valuable information to athletes, coaches, and other athletic support personnel. Specifically, conscientiousness, a personality factor, and motivation, an individual difference variable, are predictive of athletic success [1-4]. Differences in personality traits explain $20 \%$ to $45 \%$ of the variance in athletic performance [2]. Additionally, the type of motivation present in athletes can be influential in determining outcomes in sport performance [5-7].

Conscientiousness relates to one's readiness to pursue athletic success, while motivation impacts an individual's willingness to engage in the necessary behaviors [8]. The field of sport psychology has yet to

Corresponding author: Joanne E. Perry, M.S., doctoral candidate, research field: clinical sport psychology. examine the interaction and/or additive effects of motivation and conscientiousness on individual endurance sport performance. The current study simultaneously examined these variables in order to better understand the combined predictive ability of both conscientiousness and motivation on individual endurance sport performance.

Conscientiousness is one of five personality traits included in the Big Five model of personality, which represents a universal, cross-cultural structure of individual differences [9]. Conscientiousness is related to being task and goal oriented, as well as being able to delay immediate gratification. Individuals with high levels of conscientiousness are described as orderly, industrious, and disciplined [10]. On the other hand, individuals with low levels of conscientiousness are described as undisciplined, lacking attention to detail, and unreliable [10]. This trait has been positively associated with many types of performance including 

and Individual Endurance Sport Performance

occupational performance, academic performance, and athletic performance [11-17].

Conscientiousness is related to higher levels of sport achievement, better training and preparation, and greater levels of athletic success [1-4]. Specifically, research demonstrates that elite athletes have higher levels of conscientiousness when compared to non-professional athletes [1]. Additionally, conscientiousness positively predicts quality of preparation and increases the likelihood of successful performances [4]. Finally, Piedmont et al. [2] tested the direct relationship between conscientiousness and athletic performance in soccer players. Results indicated that when combined with neuroticism, this variable accounted for $23 \%$ of the variance in coach ratings of performance. Furthermore, conscientiousness independently predicted $8 \%$ of the variance in actual game statistics [2].

Self-determination theory (SDT) is a theoretical framework used to understand several components of motivation [18]. Self-determination is defined as a self-directed and flexible capacity to choose among several options to bring about desired consequences [19]. SDT examines the extent of self-determination in one's actions, in addition to the influence of internal and external forces on one's behavior [18]. Motivation is comprised of three broad components, all of which lie along the continuum of self-determination. These broader elements include intrinsic motivation (IM; highest levels of self-determination), extrinsic motivation (EM; moderate levels of self-determination), and amotivation (AM; lowest levels of self-determination) [5].

IM relates to participating in an activity for the pleasure and satisfaction derived from doing the activity. This domain includes three components: motivation to accomplish things, motivation to know things, and motivation to experience stimulation [5, 7].

EM is defined as engaging in behavior in order to attain external rewards. There are three types of EM including identification, introjection, and external regulation [7]. Identification occurs when an athlete values and judges the behavior as important; therefore, the behavior is performed for extrinsic reasons (i.e., achieving personal goals) but is internally regulated and controlled [7]. Introjection occurs when the external source of motivation has been internalized and is no longer needed to initiate behavior; therefore, the behaviors are controlled by internal emotions (i.e., guilt or anxiety) [7]. Lastly, external regulation relates to when an athlete performs in order to attain material rewards, interpersonal praise, or to comply with orders from outside people (e.g. coaches or parents) [7].

$\mathrm{AM}$ is related to the lowest levels of self-determinism and can be described as the absence of motivation [20]. This construct is associated with learned helplessness, such that athletes who are amotivated do not believe that their actions have an effect on their performance outcomes. These athletes experience feelings of inadequacy, incompetence, a lack of control, and can no longer recognize reasons why they should continue to train. Amotivated athletes may eventually decide to discontinue participation in their sport [7].

Past research has found positive relationships between both IM and EM in terms of athletic performance; meanwhile, AM is negatively associated with athletic performance [5-7]. Higher levels of self-determined motivation have been associated with successful outcomes in tennis performance [5], basketball performance [6], as well as positive training facets and preparation [7].

However, the relationship between self-determined motivation and athletic performance may be more complicated, as this relationship can be influenced by a variety of contextual factors. Cognitive evaluation theory [21] suggests that there are situational and environmental conditions that may undermine or diminish levels of intrinsic motivation by negatively impacting an individual's experience of autonomy or competence. These conditions include external events such as rewards, threats, and/or feedback [22]. Hart et 
al. [24] demonstrated that the highest performing athletes displayed lower levels of IM, higher levels of non-self-determined EM, and higher levels of AM, when compared to less successful athletes. It is possible that aspects of a highly competitive setting (i.e., a focus on winning, material rewards) may undermine an individual's intrinsic motivation [19, 23].

\subsection{Current Study}

Past literature has established significant relationships between conscientiousness and motivation, such that there is a positive relationship between conscientiousness and self-determination [24, 25]. This suggests that individuals with high levels of conscientiousness are more likely to have self-determined motivation [24]. In other words, individuals with high levels of readiness (conscientiousness) may be more likely to have a willingness (motivation) to enact behaviors related to positive sport outcomes [8].

Based on previous findings, conscientiousness and self-determined motivation are independently predictive of positive sport outcomes [1-7], and positively related to one another $[24,25]$. However, no study has examined the interaction and/or additive effects of these variables on sport performance. To date, previous research has only studied these variables separately. Therefore, the current study aimed to explore the roles of both conscientiousness and motivation in predicting individual endurance sport performance. Levels of motivation and conscientious were assessed, in addition to recent athletic performance(s) in a sample of individual endurance sport athletes.

Overall, we sought to explore the incremental validity of using both conscientiousness and motivation to predict sport performance. Additionally, based on previous literature, we hypothesized that: (1) Conscientiousness would positively predict athletic performance; (2) IM and EM would positively predict athletic performance; (3) AM would negatively predict athletic performance; (4) Conscientiousness would positively predict IM and EM; and Conscientiousness would negatively predict AM.

\section{Method}

\subsection{Participants}

Participants $(N=73)$ were enrolled in the current study if they were 18 years or older and identified as an athlete. Athletes were defined as either: (1) currently an individual sport collegiate athlete, or (2) a non-collegiate athlete competitive within the last 6 months. Collegiate athletes $(n=23)$, including cross-country runners and swimmers, were recruited from a mid-sized Division I university located in the Midwest. Non-collegiate athletes $(n=50)$ including runners, swimmers, triathletes, and rowers were recruited from the community by direct email and recruitment posts on internet forums related to swimming, running, cycling, and triathlons.

\subsection{Procedure}

Prior to the initiation of the study, researchers received approval from Saint Louis University's Institutional Review Board. Following this approval, informed consent was obtained from all participants. Researchers administered a demographic questionnaire, the International Personality Item Pool [26], the Sport Motivation Scale [7], and the Athletic Performance Subjective Rating Scale developed by the first author. An objective athletic performance score was computed for all participants based on their performance in recent competitions. Researchers attended a team practice to collect written data for the collegiate cross-country runners. Data was collected online for the remaining athletes.

All athletes provided information (i.e., distance, course difficulty, previous season time/personal best time, and current performance time) about recent performances. Collegiate athletes were asked about specific season-ending meets, which were compared to 

and Individual Endurance Sport Performance

their previous season times. Non-collegiate athletes were asked about competitions within the past 6 months and compared these events to their best times. Objective performance assessment was computed by subtracting the athlete's current performance time from their previous season time (collegiate athletes) or personal best time (non-collegiate athletes). This difference was divided by the athlete's previous season time (collegiate athletes) or personal best time (non-collegiate athletes), which yielded a percentage difference score (PDS). If an athlete's current performance time was greater (i.e., slower) than their personal best time, they received a positive PDS. On the other hand, if the athlete's current performance time was less (i.e., faster) than their best time, they received a negative PDS. In the presence of multiple events and/or performances, the average PDS was used.

\subsection{Measures}

\subsubsection{Demographic Questionnaire}

A demographic questionnaire was administered in order to determine eligibility for participants as well as the demographic composition of the study sample. It assessed for age, gender, race/ethnicity, education level, primary sport of competition, athletic scholarship awarded, athletic history, and athletic performance statistics from the previous season, in addition to overall best statistics.

\subsubsection{International Personality Item Pool (IPIP)}

The IPIP is a pool of items derived from the Big-Five personality factor markers [26-28]. For the purposes of this study, participants completed 10 items corresponding to conscientiousness, extraversion, agreeableness, neuroticism, and openness to experience. This inventory uses a five point Likert scale ranging from $1=$ Strongly Disagree to $5=$ Strongly Agree and higher scores on these subscales suggest higher levels of the construct. Internal consistency reliability estimates for these subscales range from $\alpha=0.77$ to 0.86 . With regard to convergent validity, the conscientiousness, extraversion, and neuroticism subscales have demonstrated high correlations with their respective NEO-FFI scales ( $r=$ $0.69-0.83, p<0.01)$. Furthermore, the agreeableness and openness to experience subscales have moderate correlations with the NEO-FFI subscales $(r=0.49$ and 0.59 , respectively) [29]. Item examples from this assessment include "I pay attention to details" (conscientiousness) and "I accept people as they are" (agreeableness).

\subsubsection{Sport Motivation Scale (SMS)}

The SMS assessed athletes' levels of IM, EM, and $\mathrm{AM}$ [7]. This questionnaire is a 28 -item inventory subdivided into seven subscales that assess IM to accomplish $(\alpha=0.79)$, IM to know ( $\alpha=0.73)$, IM to experience stimulation ( $\alpha=0.76)$, external regulation ( $\alpha=0.85)$, introjected regulation $(\alpha=0.73)$, identified regulation $(\alpha=0.69)$, and AM $(\alpha=0.73)$ [7]. This scale demonstrates sufficient reliability and validity for both male and female college students $(\mathrm{M}$ age $=18.4$ years $)$ over a variety of sports [7]. Test-retest reliability coefficients range from 0.58 to 0.84 [7]. Each subscale includes 4 items, to which individuals respond on a 7-point Likert $(1=$ does not correspond at all, $7=$ corresponds exactly). A total score for each subscale was obtained by adding the scores of the corresponding items. This scale provides support for the self-determination continuum [7].

2.3.4 Athletic Performance Subjective Rating Scale (APSRS)

The ASPRS is a new scale developed by the first author for the purposes of the current research study. Athletes were administered this scale for each of their reported performances. This questionnaire is comprised of 3 items along a 10-point Likert scale ( $1=$ not satisfied at all, $10=$ completely satisfied). Items assess athlete's satisfaction with individual performance (i.e., overall performance, effort, and outcome). Initial reliability measurements suggest sufficient internal consistency $(\alpha=0.89)$. Regarding convergent validity, this scale appropriately correlated 
with objective performance $(r=0.43, p<0.01)$, demonstrating that improved objective performance was associated with improvements in APSRS.

\section{Results}

\subsection{Sample Characteristics}

Participants $(N=73)$ had a mean age of 28.11 years $(S D=12.52$; range 18-65). The sample consisted of more women $(n=44)$ than men $(n=29)$. With regard to ethnicity, $93.4 \%$ identified themselves as Caucasian/White, 2.7\% as Asian/Asian-American, 1.4\% as African-American/Black, $1.4 \%$ as biracial, and $1.4 \%$ as Middle Eastern/Arab-American. The sample consisted of $56.1 \%$ runners, $19.2 \%$ swimmers, $6.8 \%$ triathletes, $2.7 \%$ rowers, and $15.1 \%$ who identified multiple individual endurance sports. Regarding education level, the sample included $17.8 \%$ freshmen, $5.6 \%$ sophomores, $16.7 \%$ juniors, $9.7 \%$ seniors, $12.3 \%$ post-baccalaureate student, and $38.4 \%$ reported not currently being enrolled in school. The start age of sport competition ranged from 5 years to 60 years $(M=$ $17.51 ; S D=10.86)$ and the years of competition ranged from less than one year to 42 years $(M=10.10 ; S D=$ 8.56). Of the collegiate athletes, $19.2 \%$ reported having an athletic scholarship $(M=29.86 \% ; S D=29.27)$. Regarding sport commitment level of the non-collegiate athletes, $10 \%$ reported training infrequently (i.e., not training every week), 4\% reported training somewhat frequently (i.e., 1 to 2 times per week), $26 \%$ reported frequently training (i.e., 3 to 4 times per week), $28 \%$ reported very frequent training (i.e., 4 to 5 times per week), 14\% reported training extremely frequently (i.e., 6 or more times per week).

\subsection{Preparatory Analyses}

Study variables were first assessed for normality and the presence of outliers. Data met these assumptions and did not require transformations. Next, ANOVAs were conducted to examine differences between collegiate and non-collegiate athletes among study variables. Results suggest that these two groups did not differ significantly on study variables (i.e., conscientiousness, facets of IM, facets of EM, AM, subjective performance, and objective performance; $p>$ 0.05). Due to these results, collegiate athlete and non-collegiate athlete groups were combined for further analyses. Pearson correlations were also conducted (Table 1).

\subsection{Primary Analyses}

Regression analyses were conducted in order to test study hypotheses. Results did not confirm the predictive ability of this conscientiousness on subjective or objective performance; $F(1,60)=0.444$, $p=0.508, F(1,53)=0.982, p=0.326$, respectively. Additionally, the three facets of IM did not significantly predict subjective or objective athletic performance, $F(3,56)=0.720, p=0.544, F(3,50)=$ $0.241, p=0.868$, respectively. Results also showed that the three facets of EM were not significant predictors of subjective or objective athletic performance, $F(3,58)$ $=0.495, p=0.687, F(3,51)=0.634, p=0.597$. However, regression analyses indicated that AM did significantly predict subjective athletic performance, $F(1,60)=4.562, p=0.037$, which suggests that $5.5 \%$ of the variance in subjective athletic performance can be explained by AM (Table 2).

Table 2 shows that AM $(\beta=-0.27)$ had a significant negative standardized regression weight, indicating that lower scores on AM were expected to have higher subjective athletic performance. However, AM failed to significantly predict objective athletic performance, $F(1,53)=1.718, p=0.196$ (Table 2) .

Results display that conscientiousness significantly predicted all three facets of IM: IM to know, $F(1,68)=$ 9.706, $p=0.003$; IM to accomplish, $F(1,69)=12.213$, $p=0.001$; IM to experience stimulation, $F(1,67)=$ 6.262, $p=0.015$. Results suggest that conscientiousness explains $11.2 \%$ of the variance in IM to know, $15.0 \%$ of the variance in IM to accomplish, and $8.5 \%$ of the variance in IM to experience stimulation. 
Table 1 Pearson Correlations of Study Variables.

\begin{tabular}{|c|c|c|c|c|c|c|c|c|c|c|c|c|}
\hline & Variables & $\mathrm{M}(\mathrm{SD})$ & 1 & 2 & 3 & 4 & 5 & 6 & 7 & 8 & 9 & 10 \\
\hline 1. & $\mathrm{C}$ & 38.71 (5.79) & -- & & & & & & & & & \\
\hline 2. & IM to $\mathrm{K}$ & $17.42(5.11)$ & $0.35^{*}$ & -- & & & & & & & & \\
\hline 3. & IM to $\mathrm{A}$ & $20.87(5.33)$ & $0.39 * *$ & $0.73 * *$ & -- & & & & & & & \\
\hline 4. & IM to $\mathrm{ES}$ & $21.24(4.94)$ & $0.29^{*}$ & $0.62 * *$ & $0.74 * *$ & -- & & & & & & \\
\hline 5. & Identified & $18.08(5.84)$ & $0.34 *$ & $0.54 * *$ & $0.64 * *$ & $0.66^{* *}$ & -- & & & & & \\
\hline 6. & Introjected & $13.46(5.61)$ & 0.20 & 0.17 & $0.37^{*}$ & $0.25^{*}$ & $0.39 * *$ & -- & & & & \\
\hline 7. & External & $13.46(5.61)$ & 0.01 & 0.22 & $0.26^{*}$ & $0.25^{*}$ & $0.49 * *$ & $0.50 * *$ & -- & & & \\
\hline 8. & $\mathrm{AM}$ & 7.04 (3.63) & $-0.27 *$ & $-0.29 *$ & $-0.33^{*}$ & $-0.25^{*}$ & -0.12 & 0.05 & $0.26^{*}$ & -- & & \\
\hline 9. & Sub Perf & $7.32(1.96)$ & 0.08 & -0.02 & 0.03 & 0.13 & 0.07 & -0.11 & 0.00 & $-0.27^{*}$ & -- & \\
\hline 10. & Obj Perf & $-1.65(8.56)$ & -0.14 & -0.03 & -0.04 & -0.012 & 0.06 & -0.14 & -0.09 & -0.18 & $0.42 * *$ & -- \\
\hline
\end{tabular}

Note. ${ }^{*}=p<0.05 ; * *=p<0.01 . \mathrm{C}=$ conscientiousness; IM to $\mathrm{K}=$ intrinsic motivation to know; IM to $\mathrm{A}=$ intrinsic motivation to accomplish; IM to ES = intrinsic motivation to experience stimulation; Identified = identified regulation; Introjected $=$ introjected regulation; External = external regulation; $\mathrm{AM}=$ amotivation; Sub Perf $=$ subjective performance; Obj Perf $=$ objective performance.

Table 2 Summary of Univariate Regression Analysis for AM Predicting Athletic Performance $(N=61)$.

\begin{tabular}{|c|c|c|c|c|}
\hline Variable & $B$ & $S E B$ & $\beta$ & $p$ \\
\hline \multicolumn{5}{|c|}{ Regression 1} \\
\hline $\mathrm{AM}$ & -0.468 & 0.357 & -0.177 & 0.196 \\
\hline \multicolumn{5}{|c|}{ Regression 2} \\
\hline $\mathrm{AM}$ & -0.145 & 0.068 & -0.266 & 0.037 \\
\hline
\end{tabular}

Note. Regression 1. DV: Objective performance; Adj $R^{2}=0.013$. Regression 2. DV: Subjective performance; $\operatorname{Adj} R^{2}=0.055$. AM $=$ Amotivation.

Overall, conscientiousness had significant positive standardized regression weights [IM to know $(\beta=0.35)$, IM to accomplish $(\beta=0.38)$, IM to experience stimulation $(\beta=0.29)$ ] demonstrating that higher scores on conscientiousness were expected to have higher scores on the three facets of IM.

Regarding EM, conscientiousness significantly predicted identified regulation, $F(1,69)=8.985, p=$ 0.004 , suggesting that conscientiousness explains $11.5 \%$ of the variance in identified regulation. Conscientiousness had a positive standardized regression weight $(\beta=0.34)$, suggesting that higher scores on conscientiousness were expected to have higher scores on identified regulation. Alternatively, conscientiousness failed to significantly predict introjected regulation and external regulation $[F(1,69)$ $=2.938, p=0.091 ; F(1,69)=0.004, p=0.952$, respectively].

Conscientiousness significantly predicted AM, $F(1$, $69)=5.19, p=0.026$, demonstrating that $5.7 \%$ of the variance in AM can be explained by conscientiousness. Conscientiousness had a significant negative standardized regression weight $(\beta=-0.27)$, indicating that higher scores on conscientiousness were expected to have lower scores on AM.

\section{Discussion}

The current study sought to explore the combined predictive ability of conscientiousness and motivation on individual endurance sport performance. Results demonstrated that $\mathrm{C}$, IM, and EM did not significantly predict subjective athletic performance. Therefore, researchers were unable to comment on the incremental validity of using both conscientiousness and motivation to predict sport performance.

The hypothesis that AM would predict decreased subjective performance was supported by the current study. This finding suggests that AM is a more relevant construct when predicting performance in a heterogeneous sample of athletes. Overall, these 

and Individual Endurance Sport Performance

findings demonstrate that IM and EM do not positively predict athletic performance; however, the absence of motivation (i.e., AM) plays a deleterious role. In other words, motivation is necessary but not sufficient for successful athletic performance.

AM, or the absence of motivation, refers to athletes who do not believe their actions have an effect on performance outcomes and has been related to decreased athletic performance [5-7]. AM may be of particular interest due to its association with athlete burnout. Athlete burnout is a condition that is characterized by physical and emotional exhaustion, reduced personal accomplishment, and sport devaluation [30]. This construct has been linked to negative performance consequences, decreased well-being, and may result in the discontinuation of sport [31]. Burned out athletes generally show a shift from a self-determined involvement in sport to a lesser degree of self-determination, the extreme of which is AM [31-33]. Furthermore, AM has demonstrated significant positive correlations with the three components of burnout including physical and emotional exhaustion $(r=0.55)$, reduced accomplishment $(r=0.52)$, and sport devaluation $(r=$ 0.71). Research has suggested that the expression of $\mathrm{AM}$ is equivalent to sport devaluation; in other words, individuals stop valuing their involvement in athletics due to the feeling that efforts will not yield a desirable result [20].

Our findings regarding the predictive ability of IM and EM on subjective athletic performance were not consistent with previous literature [7]. In our sample these variables are less relevant for predicting athletic success. However, it is also important to consider the effects on subjective performance ratings due to the heterogeneity of athletic commitment in the sample. The collegiate and non-collegiate groups may have had different expectations of their performance(s), which could have differentially affected their self-reported satisfaction with races. In other words, these groups may have different standards of success; being "completely satisfied" for a non-collegiate athlete could have a different meaning when compared to a collegiate athlete. Past research has used coach's ratings of athlete performance, as opposed to athlete self-evaluation, which may protect against the effects of expectations on self-reported satisfaction [2]. Future studies may want to incorporate coach's ratings.

Past research has also demonstrated that individuals with high levels of conscientiousness are more likely to have self-determined motivation [24, 25]. In support of these claims, conscientiousness significantly predicted all three facets of IM, as well as the facet of extrinsic motivation with the highest level of self-determinism (i.e., identified regulation). This suggests that high levels of conscientiousness predicted high levels of self-determined motivation in our sample, which was expected. Alternatively, high levels of conscientiousness were predictive of low levels of AM (the least self-determined type of motivation). Conscientiousness is related to being task and goal oriented, which is somewhat contrary to the learned helplessness observed in AM [7, 10]. These results could be important when attempting to identify athletes at risk for burnout; in other words, conscientiousness may serve as a protective factor for this condition.

Research has demonstrated positive relationships between conscientiousness and subjective athletic performance $[1,2,4]$; however, contrary to these findings, the current study did not reveal conscientiousness as a significant predictor of subjective athletic performance. These results suggest that conscientiousness may not be a salient factor when predicting individual sport performance. However, this finding may have been due to the heterogeneity of the sample's expectations of performance (as previously discussed). Furthermore, the current study used a general inventory of conscientiousness, as opposed to a sport-specific measure. It is possible that athletes display differing levels of conscientiousness between sport and daily life requirements. Thus, it may be beneficial for future research to develop a 

and Individual Endurance Sport Performance

conscientiousness scale specific to the athletic domain.

Regarding objective athletic performance, research has supported conscientiousness, IM, EM, and AM as significant predictors $[2,5,6]$; however, results from the current study did not confirm these findings. This discrepancy in results might be due to limitations of the dependent variable (i.e., objective athletic performance). It is likely that previous studies included a more consistent way of evaluating objective athletic performance across competitive situations, such as game or season statistics $[2,5,6]$. In the current study, objective athletic performance was calculated by comparing the current time to a previous race (i.e., best time or previous season time). Unfortunately, the non-collegiate group's times were compared to their best times, as opposed to previous season times. Additionally, runners and triathletes made up $62 \%$ of the non-collegiate group. When considering these sports, it is important to note that course difficulty and race day conditions vary considerably. Therefore athletes may have had a successful race; however, due to high course and condition difficulty, their current times would not accurately reflect their performance. In support of this explanation, objective athletic performance varied considerably more for non-collegiate athletes $(S D=10.49)$ compared to collegiate athletes $(S D=1.99)$. This variable would be better used in a study with a homogeneous sample regarding athletic commitment and consistent training. It is also necessary to compare races that were completed in consistent competitive environments (i.e., weather conditions, course difficulty, and time of season).

\subsection{Clinical Implications}

The study results provide support for the detrimental role of AM on athletic performance. An applied example of this relationship may be the established association between AM and burnout. High levels of $\mathrm{AM}$ are related to high levels of athlete burnout, which is characterized by decreases in performance $[20,30]$.
Burnout prevention has been associated with stress management, periodized training, recovery, nutrition, increases in coping resources, increases in social support, optimism, and adaptive coaching styles [31, 34-36]. While research has established preventative measures for burnout, fewer studies have examined treatment options for athletes already experiencing burnout. Jouper et al. [37] published a case study supporting a mindfulness and Qigong exercise program as a treatment for burnout. This may be a beneficial approach; however, it is necessary to explore other treatment options. Given the strong associations between $\mathrm{AM}$ and burnout, interventions aimed at increasing motivation may serve as an appropriate treatment approach. Motivational interviewing is an intervention aimed at increasing intrinsic motivation for behavior change, in addition to exploring and resolving client ambivalence [38]. This approach is guided by four principles including expressing empathy, supporting self-efficacy, rolling with resistance, and developing discrepancy [38]. While this technique is often used for health behavior change, it may be a beneficial option for burned out athletes [39]. For example, developing discrepancy between current practice behaviors and desired practice behaviors could serve as a way to increase motivation for change. While it is still unclear whether changes in motivation are a cause or a symptom of burnout, it appears that, either way, this aspect is important to address clinically [31].

\subsection{Limitations}

The findings from the present study are not to be interpreted without consideration of several limitations. As mentioned before, the objective measure of performance was likely limited by the variability in race conditions. The subjective measure may have been limited by differences in expectations for performance between the collegiate and non-collegiate group. The current study was also limited by the general measure of conscientiousness. Results may have had more validity if a sport-specific measure of this construct had 
been distributed. Finally, this study was limited by including athletes from different tiers of success. According to Silva's personality-performance pyramid, as athletes increase in success, they decrease in personality heterogeneity [40]. This may have contributed to the inconsistent findings in the current study compared to past research, which has largely been focused on elite athletes.

\section{Conclusions}

The results of the current study suggest that motivation is necessary but not sufficient for successful athletic performance. This is evident by the absence of significant relationships between IM, EM, and athletic performance, in addition to the predictive ability of AM for unsuccessful performance. The deleterious effect of AM on athletic performance is an important finding, especially when considering the relationship between AM and burnout. This is an important area for future research, particularly among collegiate athletes. Research suggests that collegiate athletes are at risk for experiencing burnout due to their high levels of personal stress (i.e., balancing class, practice, personal life, and regular competition [31]). Additionally, this population may be easier to reach for burnout intervention programs, compared to elite or professional athletes, given their presence on campus and involvement within athletic departments.

The predictive ability of conscientiousness on athletic performance was not supported in the current study; however, it may be valuable for future research to explore this hypothesis using a homogeneous sample of elite athletes with a sport-specific measure of conscientiousness. Further investigation of this variable may provide relevant information regarding factors contributing to athletic success.

\section{References}

[1] Ghaderi, D., and Ghaderi, M. 2012. "Survey the Relationship between Big Five Factor, Happiness and Sport Achievement in Iranian Athletes." Annals of Biological Research 3 (1): 308-12.
[2] Piedmont, R., Hill, D., and Blanco, S. 1999. "Predicting Athletic Performance Using the Five-Factor Model of Personality." Personality and Individual Differences 27 (4): 769-77.

[3] Renfrow, N. E., and Bolton, B. B. 1981. "Physiological and Psychological Characteristics Associated with Women's Participation in Intercollegiate Athletics." Perceptual and Motor Skills 53 (1): 90.

[4] Woodman, T., Zourbanos, N., Hardy, L., Beattie, S., and McQuillan, A. 2010. "Do Performance Strategies Moderate the Relationship between Personality and Training Behaviors? An Exploratory Study.” Journal of Applied Sport Psychology 22 (2): 183-97.

[5] Gillet, N., Berjot, S., and Gobance, L. 2009. "A Motivational Model of Performance in the Sport Domain." European Journal of Sport Science 9 (3): 151-8.

[6] McAuley, E., and Tammen, V. V. 1989. "The Effects of Subjective and Objective Competitive Outcomes on Intrinsic Motivation." Journal of Sport \& Exercise Psychology 11 (1): 84-93.

[7] Pelletier, L. G., Fortier, M. S., Vallerand, R. J., Tuson, K. M., and Brière, N. M. 1995. "Toward a New Measure of Intrinsic Motivation, Extrinsic Motivation, and Amotivation in Sports: The Sport Motivation Scale (SMS)." Journal of Sport and Exercise Psychology 17 (1): 35-53.

[8] Di Domenico, S. I., and Fournier, M. A. 2015. "Able, Ready, and Willing: Examining the Additive and Interactive Effects of Intelligence, Conscientiousness, and Autonomous Motivation on Undergraduate Academic Performance." Learning and Individual Differences 40: 156-62.

[9] Costa, P. T., and McCrae, R. R. 1995. "Solid Ground in the Wetlands of Personality: A Reply to Block." Psychological Bulletin 117 (2): 216-20.

[10] Hampson, S. E. 2012. "Personality Processes: Mechanisms by Which Personality Traits "Get Outside the Skin”." Annual Review of Psychology 63: 315-39.

[11] Barrick, M. R., Mount, M. K., and Strauss, J. P. 1993. "Conscientiousness and Performance of Sales Representatives: Test of the Mediating Effects of Goal Setting." Journal of Applied Psychology 78 (5): 715-22.

[12] Bidjerano, T., and Dai, D. Y. 2007. "The Relationship between the Big-Five Model of Personality and Self-regulated Learning Strategies." Learning and Individual Differences 17: 69-81.

[13] De Feyter, T., Caers, R., Vigna, C., and Berings, D. 2012. "Unraveling the Impact of the Big Five Personality Traits on Academic Performance: The Moderating and Mediating Effects of Self-efficacy and Academic Motivation." Learning and Individual Differences 22 (4): 439-48. 

and Individual Endurance Sport Performance

[14] Komarraju, M., Karau S. J., and Schmeck, R. 2009. "Role of the Big Five Personality Traits in Predicting College Students' Academic Motivation and Achievement." Learning and Individual Differences 19 (1): 47-52.

[15] Phillips, P., Abraham, C., and Bond, R. 2003. "Personality, Cognition, and University Students' Examination Performance." European Journal of Personality 17 (6): 435-48.

[16] Richardson, M., and Abraham, C. 2009. "Conscientiousness and Achievement Motivation Predict Performance.” European Journal of Personality 23 (7): 589-605.

[17] Watanabe, S., Tareq, M., and Kanazawa, Y. 2011. "When Openness to Experience and Conscientiousness Affect Continuous Learning: A Mediating Role of Intrinsic Motivation and a Moderating Role of Occupation." Japanese Psychological Research 53 (1): 1-14.

[18] Deci, E. L., and Ryan, R. M. 1985. Intrinsic Motivation and self-Determination in Human Behavior. New York: Plenum.

[19] Chantal, Y. Y., Guay, F. F., Dobreva-Martinova, T. T., and Vallerand, R. J. 1996. "Motivation and Elite Performance: An Exploratory Investigation with Bulgarian Athletes." International Journal of Sport Psychology 27 (2): 173-82.

[20] Holmberg, P., and Sheridan, D. A. 2013. "Self-determined Motivation As a Predictor of Burnout among College Athletes." Sport Psychologist 27 (2): 177-87.

[21] Deci, E. L. 1975. Intrinsic Motivation. New York: Plenum. doi:10.1016/j.lindif.2012.03.013.

[22] Ryan, R. M., and Deci, E. L. 2000. "Intrinsic and Extrinsic Motivations: Classic Definitions and New Directions." Contemporary Educational Psychology 25 (1): 54-67.

[23] Fortier, M. S., Vallerand, R. J., Briere, N. M., and Provencher, P. 1995. "Competitive and Recreational Sport Structures and Gender: A Test of Their Relationship with Sport Motivation." International Journal of Sport Psychology 26: 24-39.

[24] Hart, J. W., Stasson, M. F., Mahoney, J. M., and Story, P. 2007. "The Big Five Personality Traits and Achievement Motivation: Exploring the Relationship between Personality and Two-factor Model of Motivation." Individual Differences Research 5 (4): 267-74.

[25] Ingledew, D. K., Markland, D. A., and Sheppard, K. E. 2004. "Personality and Self-determination of Exercise Behaviour." Personality and Individual Differences 36 (8): 1921-32.

[26] Goldberg, L. R. 1999. “A Broad-bandwidth, Public Domain, Personality Inventory Measuring the Lower Level Facets of Several Five-factor Models." Personality Psychology in Europe 7: 7-28.

[27] Goldberg, L. R. 1992. "The Development of Markers for the Big-Five Factor Structure." Psychological Assessment
4 (1): 26-42.

[28] Goldberg, L. R., Johnson, J. A., Eber, H. W., Hogan, R., Ashton, M. C., Cloninger, C. R., and Gough, H. G. 2006. "The International Personality Item Pool and the Future of Public-domain Personality Measures." Journal of Research in Personality 40 (1): 84-96.

[29] Gow, A. J., Whiteman, M. C., Pattie, A., and Dreary, I. J. 2005. 'Goldberg's 'IPIP' Big-Five Factor Markers: Internal Consistency and Concurrent Validation in Scotland." Personality and Individual Differences 39 (2): 317-29.

[30] Raedeke, T. D., and Smith, A. L. 2001. "Development and Preliminary Validation of an Athlete Burnout Measure." Journal of Sport and Exercise Psychology 23: 281-306.

[31] Gould, D., and Whitley, M. A. 2009. "Sources and Consequences of Athletic Burnout among College Athletes." Journal of Intercollegiate Sport 2 (1): 16-30.

[32] Cresswell, S. L., and Ekiund, R. C. 2005b. "Motivation and Burnout among Top Amateur Rugby Players." Medicine and Science in Sports and Exercise 37 (3): 469-77.

[33] Lemyre, P., Roberts, G. C., and Stray-Gundersen, J. 2007. "Motivation, Overtraining, and Burnout: Can Self-determined Motivation Predict Overtraining and Burnout in Elite Athletes?" European Journal of Sport Science 7 (2): 115-26.

[34] Altahayneh, Z. 2013. "The Relationship between Perceived Coaches' Leadership Behaviors and Athletes' Burnout in Jordan." International Journal of Academic Research 5 (1): 60-5.

[35] Gustafsson, H., and Skoog, T. 2012. "The Mediational Role of Perceived Stress in the Relation between Optimism and Burnout in Competitive Athletes." Anxiety, Stress \& Coping 25 (2): 183-99.

[36] Tabei, Y., Fletcher, D., and Goodger, K. 2012. "The Relationship between Organizational Stressors and Athlete Burnout in Soccer Players." Journal of Clinical Sport Psychology 6 (2): 146-65.

[37] Jouper, J., and Gustafsson, H. 2013. "Mindful Recovery: A Case Study of a Burned-out Elite Shooter."Sport Psychologist 27 (1): 92-102.

[38] Miller, W. R. 1995. Motivational Enhancement Therapy Manual: A Clinical Research Guide for Therapists Treating Individuals with Alcohol Abuse and Dependence, by William, R. M., and Rockville, Md. (6000 Executive Boulevard, Rockville 20892-7003): U.S. Dept. of Health and Human Services, Public Health Service, National Institutes of Health, National Institute on Alcohol Abuse and Alcoholism.

[39] Burke, B. L., Arkowitz, H., and Menchola, M. 2003. "The Efficacy of Motivational Interviewing: A Meta-analysis of Controlled Clinical Trials." Journal of Consulting and 

and Individual Endurance Sport Performance

Clinical Psychology 71 (5): 843-61.

[40] Cox, R. H. 2011. Sport Psychology: Concepts and
Application (7th Edition). McGraw-Hill Publishing Company. 